\title{
Curvatura de la Luz
}

\author{
JAVIER SÁNCHEZ ViLlanUEVA
}

Escuela de Física - UNAH, mail: rokapier@gmail.com

Recibido: 16 de Septiembre de 2015 / Aceptado: 10 de Noviembre de 2015

\begin{abstract}
Resumen
The idea that light may be bent gravitationally when passing near massive bodies is old, stemming at least back to Newton and Laplace. In a corpuscular model of light, such as the one of Newton, it is natural that the gravitational attraction will make an otherwise straight light path bend like the trajectory of any material body. The gravitational bending of light by the Sun computed in Newtonian theory for a massive photon, with the limit of the mass going to zero, turns out to be 0.87 arcseconds - exactly one half of the value predicted by general relativity. When Eddington measured the true value during a Solar eclipse observed from the island of Principe in 1919, he obtained a result which agreed with that of Einstein's theory within errors bars, whereas the Newtonian prediction was a factor of 2 too small. It was this success ("Newton was wrong - Einstein was right") that brought world fame Einstein. Today the general relativistic value of the deflection angle has been proven correct to the per cent level.
\end{abstract}

Keywords: Corpuscular Model of Light, General Theory of Relativity, Euclidean Space, Tensor, Schwarzchild Metric, Covarian Derivative, Geodesic.

La idea de que la luz se curva cuando pasa cerca de un cuerpo masivo es vieja, se remonta al menos a Newton y Laplace. En un modelo corpuscular de la luz, tal como el de Newton, es natural que la atracción gravitacional hará que una línea recta de luz se curve como la trayectoria de cualquier cuerpo de materia. El doblamiento gravitacional de la luz debido al sol vista en la teoría de Newton para un fotón masivo, tomando que la masa tiende a cero, resulta ser 0.87 arcosegundos - exactamente la mitad del valor predicho por la teoría general de la relatividad. Cuando Eddington midió el valor correcto durante un eclipse solar observado en la isla del príncipe en 1919, obtuvo un resultado en acuerdo con la teoría de Einstein, donde la predicción de Newton fue de un factor de 2 demasiado pequeño. Fue este suceso ("Newton se equivocó - Einstein tuvo razón") que trajo fama a Einstein. Hoy el valor relativístico del ángulo deflectado se ha provado correctamente en varias observaciones a un valor exacto.

Palabras claves: Modelo Corpuscular de la Luz, Teoría General de la Relatividad, Espacio Euclídeo, Tensor, Métrica de Schwarzchild, Derivada Covariante, Geodésica.

\section{ECUACIÓN DE MOVIMIENTO}

Tratemos el concepto de corrimiento paralelo (o traslacional) de un vector. En un espacio no ecuclídeo, la traslación infinitesimal de un vector se define como un corrimiento en el que las componentes del mismo no cambian en un sistema de coordenadas que es galileano en el elemento de volumen infinitesimal dado. Para más detalle refiérase a [6].

Si $x^{\sigma}=x^{\sigma}(s)$ es la ecuación paramétrica de una curva [9] (s es longitud de arco contada a partir de un cierto punto), el 4-vector $k^{\sigma}=d x^{\sigma} / d s$ es un vector unitario tangente a la curva (Ver Figura 1). Si la curva que estamos considerando es una geodésica, a lo largo de ella $D k^{\sigma}=0$ (ver ecuación (1) que se muestra más adelante). Esto significa que si el vector $k^{\sigma}$ se somete a una traslación desde el punto $x^{\sigma}$ de una curva geodésica al punto $x^{\sigma}+d x^{\sigma}$ de la misma, coincide entonces con el vector $k^{\sigma}+d k^{\sigma}$ tangente a la curva en el punto $x^{\sigma}+d x^{\sigma}$. Cuando la tangente a una geodésica se mueve a lo largo de una curva se desplaza paralelamente a si misma $[6]$.
Por otra parte, en la traslación de dos vectores, el ángulo que forman entre ellos se debe conservar. Para más detalle refiérase a [10]. Por lo tanto, podemos decir que en la traslación de cualquier vector a lo largo de una curva geodésica, el ángulo formado por el vector y la tangente a la misma no varía. En otras palabras, en la traslación de un vector su componente a lo largo de una geodésica debe ser la misma en todos los puntos de la trayectoria 6.

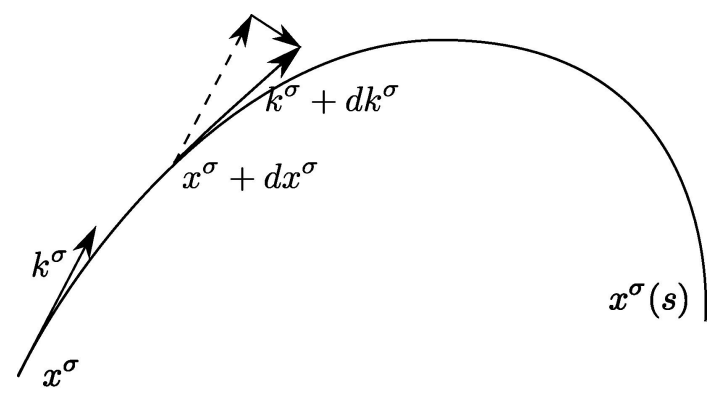

Figura 1: Transporte paralelo del 4-vector $k^{\sigma}$, desde el punto $x^{\sigma}$ hasta el punto $x^{\sigma}+d x^{\sigma}$ 
Sometamos el vector $k^{\sigma}$ a una traslación hasta el punto $x^{\sigma}+d x^{\sigma}$; el cambio que experimenta en este proceso lo representaremos por $\delta k^{\sigma}$. La diferencia $D k^{\sigma}$ entre los dos vectores, que están ahora aplicados al mismo punto, es entonces, 6]

$$
D k^{\sigma}=d k^{\sigma}-\delta k^{\sigma}
$$

donde $\delta k^{\sigma}$ y $d k^{\sigma}$ [6] son como sigue:

$$
d k^{\sigma}=\frac{d k^{\sigma}}{d x^{\nu}} d x^{\nu} \quad \mathbf{y} \quad \delta k^{\sigma}=-\Gamma_{\mu \nu}^{\sigma} k^{\mu} d x^{\nu}
$$

donde $\Gamma_{\mu \nu}^{\sigma}$ son los símbolos de Christoffel [10], que son ciertas funciones que dependen de la derivada del tensor métrico [6].

Substituyendo (2) en (1) tenemos

$$
D k^{\sigma}=\left(\frac{d k^{\sigma}}{d x^{\nu}}+\Gamma_{\mu \nu}^{\sigma} k^{\mu}\right) d x^{\nu}
$$

La expresión entre paréntesis de ecuación (3), es un tensor y se conoce como derivada covariante del vector $k^{\sigma}$ [10, 4]. Como $D k^{\sigma}=0$, tenemos que,

$$
d k^{\sigma}+\Gamma_{\mu \nu}^{\sigma} k^{\mu} d x^{\nu}=0
$$

Ahora dividiendo esta ecuación por $d s$ llegamos a la ecuación de movimiento o ecuación de la recta. En un espacio euclídeo cualquier línea recta es una geodésica [6].

$$
\frac{d^{2} x^{\sigma}}{d s^{2}}+\Gamma_{\mu \nu}^{\sigma} \frac{d x^{\mu}}{d s} \frac{d x^{\nu}}{d s}=0
$$

\section{PRopagación DE UNA SEÑAl LUMinosa}

La ecuación de movimiento (5) no es aplicable a la propagación de una señal luminosa, debido a que $d s=0$ 8. Para obtener las ecuaciones de movimiento en una forma adecuada a este caso, nos apoyaremos en el hecho de que la dirección de propagación de un rayo luminoso en la óptica geométrica se determina mediante el vector de onda, que es tangente al rayo. Podemos, por consiguiente, escribir el vector de onda cuadridimensional en la forma $k^{\sigma}=d x^{\sigma} / d \lambda$, donde $\lambda$ es un cierto parámetro que varía a lo largo del rayo. En la teoría de la relatividad especial, el vector de onda no varía a lo largo del mismo en la propagación de la luz en el vacío, esto es, $d k^{\sigma}=0$. La ecuación (5) se escribe [6]

$$
\frac{d^{2} x^{\sigma}}{d \lambda^{2}}+\Gamma_{\mu \nu}^{\sigma} \frac{d x^{\mu}}{d \lambda} \frac{d x^{\nu}}{d \lambda}=0
$$

\section{ECUACIÓN DE MOVIMIENTO PARA UN FOTÓN}

Si queremos investigar la curvatura de la luz causada por un cuerpo simétrico esférico, debemos utilizar la solución de Schwarzchild [2, 3]

$$
\begin{aligned}
d s^{2}= & (1+2 \varphi(r)) d t^{2}-(1+2 \varphi(r))^{-1} d r^{2} \\
& -r^{2}\left(d \theta^{2}+\sin ^{2} \theta d \phi^{2}\right)
\end{aligned}
$$

donde $2 \varphi(r)=-2 G M / r=-r_{S} / r$, con $\varphi(r)$ el potencial gravitacional newtoniano, $r_{S}$ el radio de Schwarzchild y $M$ la masa que genera la curvatura determinada por la métrica de Schwarzchild.

Como estamos trabajando con simetrías esféricas, podemos hacer que el rayo de luz se mueva en un ángulo constante $\theta=\pi / 2$; o sea, en el plano ecuatorial, lo cual nos queda [4]

$$
d s^{2}=\left(1-\frac{r_{S}}{r}\right) d t^{2}-\left(1-\frac{r_{S}}{r}\right)^{-1} d r^{2}-r^{2} d \phi^{2}
$$

y para las distintas coordenadas tenemos el tensor métrico $g_{i j}$ en forma covariante y contravariante, ya que

$$
\begin{aligned}
d s^{2} & =g_{i j} d x^{i} d x^{j} \\
& =g_{00} d x^{0} d x^{0}+g_{11} d x^{1} d x^{1}+g_{22} d x^{2} d x^{2}
\end{aligned}
$$

escribiendo $g_{00}=g_{t t}, g_{11}=g_{r r}$ y $g_{22}=g_{\phi \phi}[3]$

$$
\begin{aligned}
g_{t t} & =\left(1-\frac{r_{S}}{r}\right), \quad g_{r r}=-\left(1-\frac{r_{S}}{r}\right)^{-1}, \\
g_{\phi \phi} & =-r^{2} \\
g^{t t} & =\left(1-\frac{r_{S}}{r}\right)^{-1}, \quad g^{r r}=-\left(1-\frac{r_{S}}{r}\right), \\
g^{\phi \phi} & =-\frac{1}{r^{2}}
\end{aligned}
$$

Con el tensor métrico y teniendo en cuenta que $g_{i j, k}=$ $d g_{i j} / d x^{k}$, encontramos los símbolos de Christoffel [5]

$$
\begin{aligned}
\Gamma_{\mu \nu}^{\sigma} & =\frac{1}{2} g^{\sigma \beta}\left(g_{\mu \beta, \nu}+g_{\beta \nu, \mu}-g_{\mu \nu, \beta}\right) \\
\Gamma_{t r}^{t} & =\frac{r_{S}}{2 r^{2}\left(1-\frac{r_{S}}{r}\right)} \\
\Gamma_{r r}^{r} & =-\frac{r_{S}}{2 r^{2}\left(1-\frac{r_{S}}{r}\right)} \\
\Gamma_{t t}^{r} & =\frac{r_{S}}{2 r^{2}\left(1-\frac{r_{S}}{r}\right)} \\
\Gamma_{\phi \phi}^{r} & =-r\left(1-\frac{r_{S}}{r}\right) \\
\Gamma_{\phi r}^{\phi} & ==\frac{1}{r}
\end{aligned}
$$

De la ecuación de movimiento (6) para $x^{0}=t, x^{1}=r$, $x^{2}=\phi[10$

$$
\frac{d^{2} r}{d \lambda^{2}}+\Gamma_{t t}^{r}\left(\frac{d t}{d \lambda}\right)^{2}+\Gamma_{r r}^{r}\left(\frac{d r}{d \lambda}\right)^{2}+\Gamma_{\phi \phi}^{r}\left(\frac{d \phi}{d \lambda}\right)^{2}=0
$$




$$
\begin{aligned}
& \frac{d^{2} t}{d \lambda^{2}}+\Gamma_{t r}^{t} \frac{d t}{d \lambda} \frac{d r}{d \lambda}+\Gamma_{r t}^{t} \frac{d r}{d \lambda} \frac{d t}{d \lambda}=0 \\
& \frac{d^{2} t}{d \lambda^{2}}+\frac{r_{S}}{r^{2}\left(1-\frac{r_{S}}{r}\right)} \frac{d t}{d \lambda} \frac{d r}{d \lambda}=0 \\
& \frac{d^{2} \phi}{d \lambda^{2}}+\Gamma_{\phi r}^{\phi} \frac{d \phi}{d \lambda} \frac{d r}{d \lambda}+\Gamma_{r \phi}^{\phi} \frac{d r}{d \lambda} \frac{d \phi}{d \lambda}=0 \\
& \frac{d^{2} \phi}{d \lambda^{2}}+\frac{2}{r} \frac{d \phi}{d \lambda} \frac{d r}{d \lambda}=0
\end{aligned}
$$

Podemos obtener $(d r / d \lambda)^{2}$ de la métrica de Schwarzchild (8), teniendo en cuenta que $\lambda$ es el parámetro que varía a lo largo del rayo [10]

$$
\begin{aligned}
\left(\frac{d r}{d \lambda}\right)^{2}= & \left(1-\frac{r_{S}}{r}\right)+\left(1-\frac{r_{S}}{r}\right)^{2}\left(\frac{d t}{d \lambda}\right)^{2} \\
& -r^{2}\left(1-\frac{r_{S}}{r}\right)\left(\frac{d \phi}{d \lambda}\right)^{2}
\end{aligned}
$$

Haciendo el siguiente reemplazo

$$
\begin{gathered}
\frac{d r}{d \lambda}=\frac{d r}{d \phi} \frac{d \phi}{d \lambda} \\
\frac{d^{2} r}{d \lambda^{2}}=\frac{d r}{d \phi} \frac{d^{2} \phi}{d \lambda^{2}}+\frac{d^{2} r}{d \phi^{2}}\left(\frac{d \phi}{d \lambda}\right)^{2}
\end{gathered}
$$

Integrando las ecuaciones (13) y (14)

$$
\frac{d t}{d \lambda}=\frac{a}{\left(1-\frac{r_{S}}{r}\right)}, \quad \frac{d \phi}{d \lambda}=\frac{h}{r^{2}}
$$

donde $a$ y $h$ son constantes de integración [10].

Substituyendo (15), (17) y (18) en (12), encontramos la ecuación de movimiento del fotón [10]

$$
\left(\frac{d^{2} r}{d \phi^{2}}-\frac{2}{r}\left(\frac{d r}{d \phi}\right)^{2}+\frac{3}{2} r_{S}-r\right) \frac{h^{2}}{r^{4}}-\frac{r_{S}}{2 r^{2}}=0
$$

Multiplicando (19) por $\frac{r^{4}}{h^{2}}$

$$
\frac{d^{2} r}{d \phi^{2}}-\frac{2}{r}\left(\frac{d r}{d \phi}\right)^{2}+\frac{3}{2} r_{S}-r-\frac{r_{S} r^{2}}{2 h^{2}}=0
$$

Haciendo el cambio de variable $\omega=1 / r$, [10, 1 ]

$$
\begin{gathered}
\frac{d r}{d \phi}=\frac{d r}{d \omega} \frac{d \omega}{d \phi}=-\frac{1}{\omega^{2}} \frac{d \omega}{d \phi}, \\
\frac{d^{2} r}{d \phi^{2}}=\frac{2}{\omega^{3}}\left(\frac{d \omega}{d \phi}\right)^{2}-\frac{1}{\omega^{2}} \frac{d^{2} \omega}{d \phi^{2}}
\end{gathered}
$$

la ecuación de la geodésica (20) para un fotón queda

$$
\begin{aligned}
& \frac{2}{\omega^{3}}\left(\frac{d \omega}{d \phi}\right)^{2}-\frac{1}{\omega^{2}} \frac{d^{2} \omega}{d \phi^{2}}-2 \omega\left(-\frac{1}{\omega^{2}} \frac{d \omega}{d \phi}\right)^{2} \\
& +\frac{3}{2} r_{S}-\frac{1}{\omega}-\frac{r_{S}}{2 h^{2} \omega^{2}}=0
\end{aligned}
$$

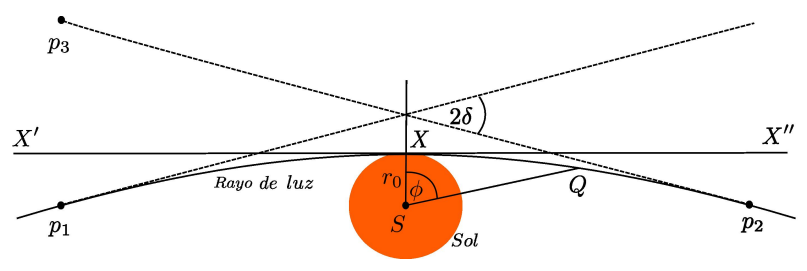

Figura 2: El rayo de luz $p_{1} X p_{2}$ se deflecta un ángulo $2 \delta$

$$
\frac{d^{2} \omega}{d \phi^{2}}-\frac{3}{2} r_{S} \omega^{2}+\omega+\frac{r_{S}}{2 h^{2}}=0
$$

Escribiendo esta ecuación (24) de la manera siguiente

$$
\frac{d^{2} \omega}{d \phi^{2}}+\omega=\frac{3}{2} r_{S} \omega^{2}-\frac{r_{S}}{2 h^{2}}
$$

y comparando esta ecuación (25) con la ecuación de movimiento de Newton [11] observamos que la diferencia es la aparición del termino $\frac{3}{2} r_{S} \omega^{2}$ [1], que la podemos tratar como una perturbación [3].

\section{Deflección de la luz CAUSAda por CUERPOS MASIVOS}

De la ecuación de movimiento (25) para un fotón con $h \rightarrow \infty$ y $r_{S}=2 G M$, tenemos [1]

$$
\frac{d^{2} \omega}{d \phi^{2}}+\omega=3 G M \omega^{2}
$$

Primero tomemos el caso para $M=0$

$$
\frac{d^{2} \omega}{d \phi^{2}}+\omega=0
$$

cuya solución homogénea es

$$
\omega_{h}(\phi)=\omega_{0} \cos \phi
$$

Esta es la ecuación de la linea recta $X^{\prime} X X^{\prime \prime}$ en coordenadas polares, donde $\omega_{0}\left(=1 / r_{0}\right)$ corresponde al valor de la distancia mas cercana desde $X$ al origen $S$ y $\phi$ es cero (ver Figura 2).

Substituyendo en (28) en (26)

$$
\frac{d^{2} \omega}{d \phi^{2}}+\omega=3 G M \omega_{0}^{2} \cos ^{2} \phi
$$

cuya solución particular es

$$
\omega_{p}(\phi)=G M \omega_{0}^{2}\left(2-\cos ^{2} \phi\right)
$$

La solución total de la ecuación (26) es

$$
\omega(\phi)=\omega_{0} \cos \phi+G M \omega_{0}^{2}\left(2-\cos ^{2} \phi\right)
$$

Haciendo que $r \rightarrow \infty$, osea $\omega=0$, en (31)

$$
G M \omega_{0}^{2} \cos ^{2} \phi-\omega_{0} \cos \phi-2 G M \omega_{0}^{2}=0
$$




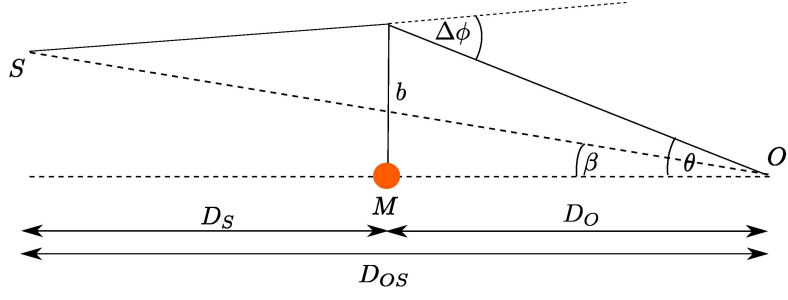

Figura 3: Geometría de una lente gravitacional

que es cuadrática en $\cos \phi$, así

$$
\cos \phi=\frac{1}{2}\left[\frac{1}{G M \omega_{0}} \pm\left(\left(\frac{1}{G M \omega_{0}}\right)^{2}+8\right)^{1 / 2}\right]
$$

Como $G M \omega_{0}$ es usualmente muy pequeño,

$\left(1 / G M \omega_{0}\right)^{2}>>8$, utilizando la expresión ,

$$
(1 \pm x)^{1 / 2}=1 \pm \frac{1}{2} x+\ldots
$$

para el término entre paréntesis elevado a la $1 / 2$ en la ecuación (33), tenemos que aproximadamente

$$
\cos \phi=\frac{1}{2}\left[\frac{1}{G M \omega_{0}} \pm \frac{1}{G M \omega_{0}}\left(1+4 G^{2} M^{2} \omega_{0}^{2}\right)\right]
$$

El signo positivo se desprecia, debido a que $\cos \phi$ no puede exceder de 1

$$
\cos \phi=-2 G M \omega_{0},
$$

o de nuevo, como $2 G M \omega_{0}$ es muy pequeño, y haciendo la siguiente relación

$$
\phi=\frac{\pi}{2}+\delta
$$

encontramos que $\delta=2 G M \omega_{0}$.

Estos resultados demuestran que la desviación neta del rayo de luz $p_{3} p_{1}$ (Figura 2) es [1, 2]

$$
2 \delta=4 G M \omega_{0}=\frac{4 G M}{r_{0}} .
$$

Esta ecuación (38) también la podemos escribir de la manera siguiente [2]

$$
\Delta \phi=\frac{4 G M}{r_{0}}
$$

\section{LENTES GRAVITACIONALES}

Ahora que conocemos la deflección causada por una masa $M$, por geometría analítica del espacio plano (o Euclideo) (ver Figura 3) encontremos la ecuación de una lente gravitacional.

Sean $D_{O}$ la distancia a la lente gravitacional, $D_{O S}$ la distancia a la fuente de luz y $D_{S}$ la distancia entre la fuente de luz y la lente gravitacional, $b$ la distancia mas corta entre el rayo de luz y la lente, $\beta$ la dirección de la fuente de luz en ausencia de una lente gravitacional, $\Delta \phi$ el ángulo deflectado y $\theta$ la dirección observada. [7]

Llamemos $L^{\prime}$ a la distancia entre el observador hasta el punto donde se deflecta el rayo y $L$ la distancia $O S$.

De la Figura 3 tenemos

$$
\begin{aligned}
& \cos \theta=\frac{D_{O}}{L^{\prime}}, \quad \sin \theta=\frac{b}{L^{\prime}} \\
& \cos \beta=\frac{D_{O S}}{L}
\end{aligned}
$$

pero debido a que $\theta$ y $\beta$ son ángulos muy pequeños

$$
\begin{aligned}
& 1=\frac{D_{O}}{L^{\prime}}, \quad \theta=\frac{b}{L^{\prime}} \\
& 1=\frac{D_{O S}}{L}
\end{aligned}
$$

Así tenemos

$$
D_{O}=L^{\prime}=\frac{b}{\theta}, \quad D_{O S}=L
$$

De la ley de los senos se puede ver que

$$
\frac{\sin (\Delta \phi-\theta+\beta)}{L^{\prime}}=\frac{\sin (180-\Delta \phi)}{L}
$$

y como $\Delta \phi, \theta$ y $\beta$ son muy pequeños

$$
\frac{\Delta \phi-\theta+\beta}{L^{\prime}}=\frac{\Delta \phi}{L}
$$

luego, con (42) en (44)

$$
\begin{gathered}
\frac{\Delta \phi-\theta+\beta}{D_{O}}=\frac{\Delta \phi}{D_{O S}} \\
(\Delta \phi-\theta+\beta) D_{O S}=\Delta \phi D_{O}
\end{gathered}
$$

despejando para $\beta D_{O S}$

$$
\beta D_{O S}=\theta D_{O S}+\Delta \phi\left(D_{O}-D_{O S}\right)
$$

de la Figura 3 vemos que $D_{O S}=D_{O}+D_{S}$, y tenemos entonces

$$
\beta D_{O S}=\theta D_{O S}-\Delta \phi D_{S}
$$

de $(38)$ con $\Delta \phi=2 \delta, r_{0}=b$, y $b=\theta D_{O}$, llegamos a la ecuación de la lente gravitacional

$$
\beta(\theta)=\theta-\frac{D_{S}}{D_{O} D_{O S}} \frac{4 G M}{\theta}
$$

esta ecuación tiene varias soluciones: esto es, el objeto fuente tiene multiples imagenes.

Veamos un caso perticular en el que $\beta=0$, osea el objeto fuente se encuentra exactamente detras de la lente gravitacional. Esto nos daría la imagen de un anillo, que 


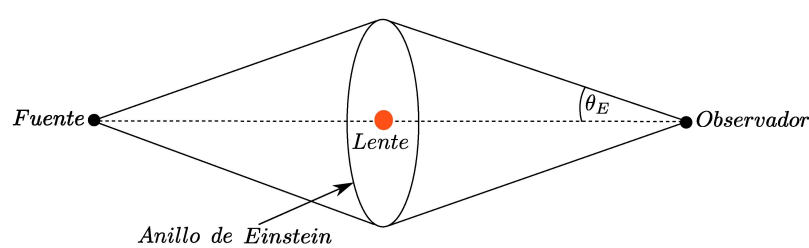

Figura 4: Anillo de Einstein formado por una masa $M$ cuando la fuente de luz se encuentra exactamente detrás de dicha masa $M$

se conoce como anillo de Einstein [7] (ver Figura 4), y cuya ecuación es

$$
\theta_{E}=\sqrt{\frac{4 G M}{c^{2}} \frac{D_{S}}{D_{O} D_{O S}}}
$$

donde anteriormente hemos venido utilizando $c=1$.

\section{Agujeros Negros}

El caso más extremo de la curvatura de la luz en un campo gravitacional ocurre en un agujero negro, objetos más pequeños que su radio de Schwarzschild, $r_{s}=2 G M$ [3].

La superficie $r=2 G M$, mientras sea localmente perfectamente regular, se conoce como un punto de no retorno - una vez que la partícula caiga en él, nunca puede salir. Se define un horizonte de eventos como una superficie donde las partículas nunca pueden escapar, y es imposible para nosotros ver en el interior, de ahí el nombre de agujero negro; este horizonte se encuentra localizado en $r=2 G M$.

Existe una analogía en la teoría de Newton para agujeros negros. La velocidad de escape Newtoniana para una partícula a una distancia $r$ de un cuerpo gravitatorio de masa $M$ es

$$
v_{\text {esc }}=\sqrt{\frac{2 G M}{r}}
$$

Si nos preguntamos ¿donde la velocidad de escape Newtoniana es igual a la velocidad de la luz?, encontramos que $r=2 G M$. Sabemos el hecho de que la rapidez de la luz no tiene un papel fundamental en la teoría de Newton, podría parecer provocativo que la luz, como una partícula inercial que se mueve a una velocidad $c$, no es capaz de escapar de un cuerpo de masa $M$ y radio menor que $2 G M$. Pero hay una profunda diferencia entre este caso y lo que vemos en la teoría general de la relatividad [3].

\section{CÁlculos y grÁficos}

Hagamos algunos cálculos para varias estrellas cercanas a la Tierra. Primero grafiquemos el ángulo $\beta$ (49) para: las estrellas Próxima Centauri y Alfa Centauri A (que pertenecen al sistema estelar Alfa Centauri) que se en-

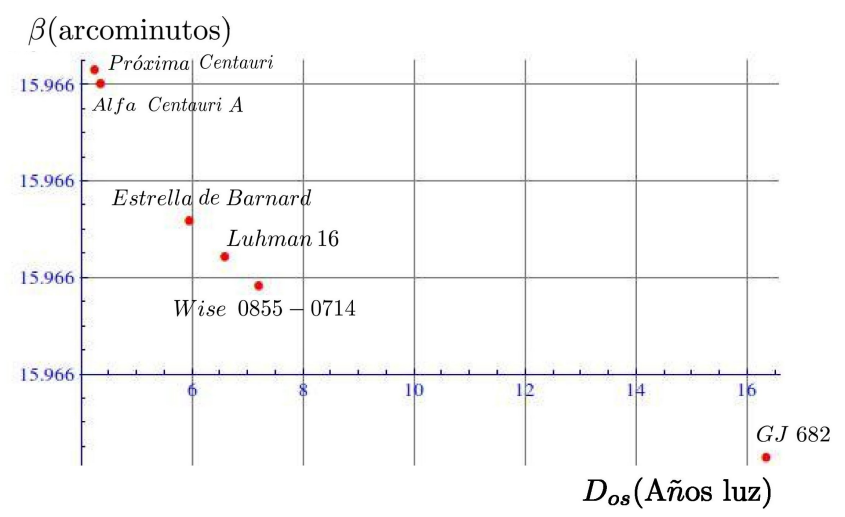

Figura 5: Ángulo $\beta$ para algunas estrellas cercanas a la Tierra

cuentran a una distancia de 4.2420 y 4.3649 lys (años luz), respectivamente.

La estrella de Barnard a 5.9629 lys, Luhman 16 a 6.59 lys, Wise 0855-0714 a 7.2 lys y GJ 682 a 16.336 lys.

Tomando en cuenta los siguientes datos

$G=6.67384 \times 10^{-11} \mathrm{~N} \cdot \mathrm{M}^{2} / \mathrm{kg}^{2}$,

$c=3 \times 10^{8} \mathrm{~m} / \mathrm{s}$ (rapidez de la luz),

$M=1.9891 \times 10^{30} \mathrm{~kg}$ (masa del Sol),

$D_{O}=1.49586 \times 10^{11} \mathrm{~m}$ (distancia Sol-Tierra),

$r=6.96 \times 10^{8} \mathrm{~m}$ (radio del Sol)

$\theta=\arctan (r / D o)=15.995139193305764$ arcominutos

(radio angular del Sol)

$p c=3.0857 \times 10^{16} \mathrm{~m}$ (parsec)

lys $=0.3066 p c$ (año luz)

$D_{O S}$ igual a la distancia de cada estrella

de la ecuación (49) encontramos estos ángulos en arcominutos

Próxima Centauri: 15.965997483263004

Alfa Centauri A: 15.965997480204624

estrella de Barnard: 15.965997451914864

Luhman 16: 15.965997444561634

Wise 0855-0714: 15.965997438637892

GJ: 15.965997402847847

En la Figura 5 se puede ver el ángulo $\beta$ (49), observando que disminuye para las estrellas más lejanas.

Calculando el ángulo $\theta_{E}(50)$ en arcosegundos para estas mismas estrellas

Próxima Centauri: 40.96403972356491

Alfa Centauri A: 40.96404187312202

estrella de Barnard: 40.964061756350446

Luhman 16: 40.964066924507215

Wise 0855-0714: 40.96407108796047

GJ: 40.96409624268024

En la Figura 6 se puede ver el ángulo $\theta_{E}$, observando que aumenta para las estrellas más lejanas. 


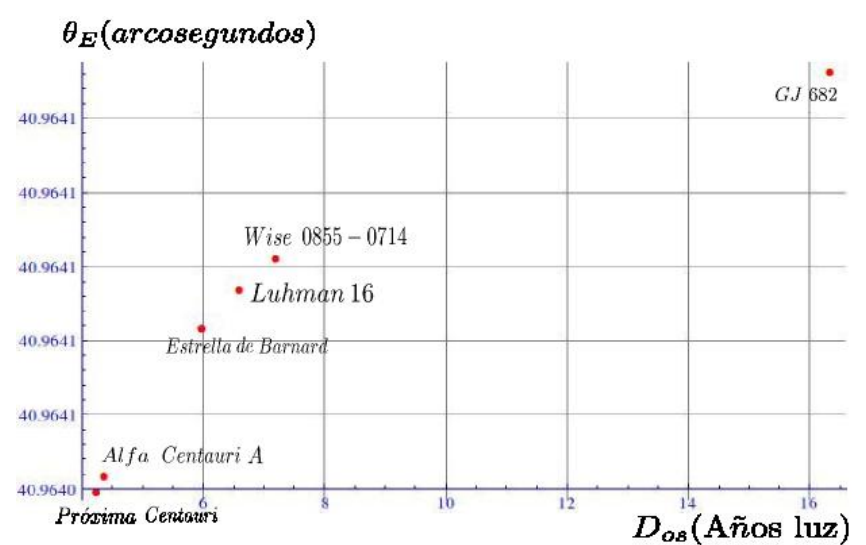

Figura 6: Ángulo $\theta_{E}$ para algunas estrellas cercanas a la Tierra

\section{CONCLUSIONES}

- En Relatividad Especial, el intervalo espacio-temporal a lo largo de la trayectoria de una partícula sin masa que se propaga a la velocidad de la luz debe ser igual a cero, $d s^{2}=0$. En Relatividad General, lo mismo debe ser cierto en cada sistema de coordenadas inercial local. Así, como el intervalo es invariante, la condición $d s^{2}=0$ sería valida a lo largo de la geodésica de la luz en cualquier espacio-tiempo. 8]

- La desviación de un rayo de luz al pasar cerca de un cuerpo masivo (M) es igual a

$$
\Delta \phi=\frac{4 G M}{r_{0}}
$$

Si $M=0$, o sea no existe un cuerpo masivo, entences el rayo viaja en línea recta sin desviarce.

- Para una estrella que se encuentra detrás del sol, la posición aparente de la estrella es desviada por 1.74 arcosegundos, de acuerdo a la teoría general de la relatividad. Este resultado fue verificado por primera vez por Eddington en 1919. [1

- Comparado a las lentes ópticas que encontramos en cámaras y binoculares, las lentes gravitacionales son muy malas si las consideramos como instrumentos ópticos, con todos los tipos de distorciones y aberraciones presente. Sin embargo son perfectamente acromáticas (producen la misma deflección de la luz para cualquier tipo de longitud de onda), lo cual es util para poder encontrarlas. La razón de esta propiedad es porque la desviación de la luz es un efecto puramente geométrico, independiente de la energía de los fotones.

\section{REFERENCIAS}

[1] A. B. A. K. Raychaudhuri, S. Banerji. General Relativity, Astrophysics, and Cosmology. Springer, Physics Department, Presidency College, Calcutta, India. Burdwan University, Burdwan, West Bengal, India. Physics Department, Jadavpur University, Calcutta 32, India, 1992.

[2] S. K. Bose. An Introduction to General Relativity. John Wiley and Sons, Inc., University of Notre Dame, Indiana, USA, 1980.

[3] S. Carroll. SpaceTime and Geometry. Addison Wesley, University of Chicago, 2004.

[4] J. A. W. Charles W. Misner, Kip S. Thorne. Gravitation. W. H. Freeman and Company, University of Maryland, California Institute of Technology, Princeton University, 1973.

[5] P. P. Jetzer. General Relativity Lecture Notes. Universität Zürich, August 17, 2011.

[6] E. M. L. L. D. Landau. Teoría Clásica, de los Campos. Academia de Ciencias, U.R.S.S, second edition, 1973.

[7] M. S. Longair. Galaxy Formation. Springer, University of Cambridge, Department of Physics, 1998.

[8] V. Mukhanov. Physical Foundations of Cosmology. Cambridge University Press, Ludwig Maximilians Universität München, 2005.

[9] A. Pressley. Elementary Differential Geometry. Department of Mathematics, King's College London, second edition, 2010.

[10] I. S. Sokolnikoff. Analisis Tensorial. Second edition, 1976.

[11] K. R. Symon. Mecánica. Aguilar, Universidad de Wisconsin, 1968. 